\title{
3D-printed Drugs: A Fabrication of Pharmaceuticals towards Personalized Medicine
}

\author{
Muhammad Salahuddin Haris ${ }^{1,2, \star}$, Nur Hakimah Mohd Azlan², Muhammad Taher ${ }^{1}$, Shaiqah Mohd Rus ${ }^{2}$, \\ Bappaditya Chatterjee ${ }^{3}$
}

1'Department of Pharmaceutical Technology, Kulliyyah of Pharmacy, International Islamic University Malaysia, Kuantan, Pahang, MALAYSIA.

${ }^{2}$ Advanced Drug Delivery Lab, Kulliyyah of Pharmacy, International Islamic University Malaysia, Kuantan, Pahang, MALAYSIA. ${ }^{3}$ Shobhaben Pratapbhai Patel School of Pharmacy \& Technology Management (SPPSPTM), SVKM's NMIMS, Mumbai, Maharashtra, INDIA.

\begin{abstract}
Background: Three-dimensional (3D) printing fabricates a structure by depositing materials layer-by-layer to form an object. Drug personalization receives attention due to problems arising from drug treatments such as undesirable side effects and ineffective drug therapy among pediatric and geriatric patients. Hypothesis: Conventional dosage form faces issues such as non-adherence to medication, ineffective treatment due to non-preferable dosage form and non-optimized drug release. Personalized 3D printing drugs offer personalized drug dosing, novel drug release profile and unique dosage form production. Results and Conclusion: Therefore, this review discussed the benefits of 3D-printed drugs and an overview of the standard 3D printer technology used in pharmaceuticals. This article aimed to review the current application of 3D-printed drugs in the personalization of drug dosing, the variation of drug release profile and fabrication of unique drug dosage form. Further research and experimentation of 3D-printed drugs are recommended as they will offer a significant aid in the customization of medicine. The potential utilization of 3D-printed drugs in optimizing effective and safe medication is undeniable. However, there are some challenges needed to be overcome, such as compatibility between chosen methods with materials used, difficulty to scale-up production, regulatory requirement as well as protection of individual rights issues.
\end{abstract}

Key words: 3D-printed drugs, Personalized dosing, 3D printer, Polypills, Patient compliance, Drug release profile.

\section{INTRODUCTION}

Three-dimensional (3D) printing or also termed as Additive manufacturing (AM) is a process in which a structure previously prototyped in software is fabricated into a structure layer-by-layer until a $3 \mathrm{D}$ object is formed. ${ }^{1,2}$ The fabricating of drugs layerby-layer with thickness ranges between 0.001 to 0.1 inches by the $3 \mathrm{D}$ printer is a unique feature that enables the modification of shapes, pattern, or fill density which consequently results in improvement of the geometric complexity of the drugs. ${ }^{3}$ The complex geometries enable the controlled release of the drugs and more tailordosing specific to the patient. ${ }^{4}$ Currently, there is an accelerating emergence of realization for applications and studies on drug delivery using this technology since the first 3D-printed orodispersible tablet Spritam $^{\circledR} \quad$ (levetiracetam) anti-epileptic drug was approved by the Food and Drug Administration (FDA) in 2015. ${ }^{1}$

The 3D printer in pharmaceuticals has sparked a growing interest as it offers some advantages over the conventional drug manufacturing method. Firstly, the implementation of 3D printing technologies in drug development are more economical for personalized drugs and small-scale drugs production. The application of
Submission Date: 18-03-2020; Revision Date: 29-05-2020; Accepted Date: 13-08-2020

DOI: 10.5530/ijper.54.3s.139 Correspondence: Dr. Muhammad Salahuddin Haris

Department of

Pharmaceutical Technology, Kulliyyah of Pharmacy, International Islamic University Malaysia (IIUM) Jalan Sultan Ahmad Shah25200, Kuantan, Pahang, MALAYSIA.

Phone: +609 5714855 E-mail: solah@iium.edu.my

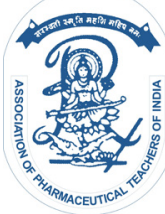

www.ijper.org 
3D-printed drugs is mainly due to the ability to produce personalized drugs such as drugs explicitly developed according to patient's weight, disease and preference of dosage forms. The preparation of these personalized drugs is more straightforward and economical using $3 \mathrm{D}$ printer compared to the conventional method to tailor and control the number of materials and active ingredients used during modification of printed drug at the design level. ${ }^{5}$ This method is also applicable for the development of small-scale drug production such as orphan drugs which is only consumed by very few patients.

The steps involved in 3D printing are designing a product using computer-aided design (CAD), product printing and post-processing such as drying or polishing the product. The development of drug formulation with different dosage doses or small scale drug production is relatively low using $3 \mathrm{D}$ printing method. ${ }^{6-8}$ In contrast, the wet granulation method as one of the conventional tableting procedures involves complex manufacturing steps, but more economical for larger-scale production. They are: (i) mixing active pharmaceutical ingredients (APIs) with fillers such as lactose, (ii) agglomeration in binder liquid, (iii) drying, (iv) milling to reduce the particle size of the powder mixture, (v) mixing the powder mixed with other excipients and lastly, (vi) tableting.

Another advantage of 3D printed drug is the potential for optimization of dosing and spatial distribution. 3D-printed drug minimizes the cost of APIs and excipients as it optimizes the dosing and spatial distribution, which is particularly beneficial for pediatric and geriatric patients. ${ }^{2,5}$ Moreover, it emerges as a better alternative to produce a narrow therapeutic window and expensive drugs. Besides, 3D printing is very advantageous when printing small batches of drug with specified dosage forms, dose and release profile. These capabilities render the fabrication of personalized drug potentially achievable using the technology.

The importance of 3D printing in personalized medicine becomes significant with issues reported on adverse drug reaction (ADR), particularly among pediatric and geriatric patients. ${ }^{10-12}$ It has been recorded that the percentage of hospital admission in England due to ADR cases increased by $53.4 \%$ from 60055 to 92114 in $2008 / 2009$ to $2014 / 2015$, respectively. ${ }^{12}$ The current application on usage and dose of a drug is based on empirical treatment, which suits many people. However, these regimens may be ineffective or even lead to undesirable side effects for others. Such outcome is attributable towards the differences in patient's background and their different metabolisms and necessities. ${ }^{13}$ Besides, there are cases in which some patients do not adhere to their prescribed drug regimen, thus resulting in an ineffective treatment. Nonadherence is a problem mainly associated with nonpreferred dosage forms and palatability of medications. In contrast, 3D printing considers a patient's requirements, namely age, gender, weight, comorbidities and pharmacokinetics of the drug to fabricate a personalized medicine. ${ }^{14}$ Dose adjustment is frequently based on empirical methods, and therefore, the chance of undesirable side effects to occur is high. 3D printing able to tailor-made drugs specific to requirements as needed by patients. Therefore, the application of 3D printing in drug fabrication to personalize the dosing, to vary the drug release and to manufacture unique drug dosage forms are great potential solutions for all problems mentioned above. This article reviews the application of 3D printing in drug fabrication to provide a possible solution for common drug problems by fabricating personalized drugs, altering drug release profile and producing unique dosage forms of drugs. Besides, this article also briefly describes the challenges or limitations faced by $3 \mathrm{D}$ printing in drug manufacturing.

\section{Basic procedure of 3D printing}

There are several types of 3D printing, namely 3D Inkjet Printing, 3D Powder Bed Printing, Selective Laser Sintering (SLS) and Fused Deposition Modelling (FDM). Regardless, all utilize the same necessary procedure as summarized in Figure 1. Firstly, the product is designed using computer-aided design (CAD) software before converted into a 3D printer readable file, which is usually in the STL file format. Then, the materials are processed to aid the printing process. Following this, the raw materials are used in printing and they are made into the desired shapes layerby-layer until the product is formed. Lastly, the product is removed, whereby some may require post-processing such as polishing and drying to be done. Any unused material can be recycled for reuse in subsequent printing cycles. ${ }^{15,16} 3 \mathrm{D}$ printing is very advantageous compared to other manufacturing methods as it can cater for more flexible dosing and complex geometries.

\section{Types of 3D printing}

The application of 3D printer in pharmaceuticals enables the fabrication of novel, unique and complex dosage forms. The 3D printers chosen for drug fabrication depend on various factors, including the characteristics of the drugs and excipients. This paper 
briefly reviewed some of the typical 3D printers used in the pharmaceutical field.

\section{D Inkjet Printing}

Inkjet printing is a type of $3 \mathrm{D}$ printing that utilizes the electromagnet, heat and piezoelectric technology to force 'ink' onto a material, i.e. polymer powder bed on the stage. Among parameters need to be taken into account for inkjet printing are viscosity and rheological properties of the ink. An ink with viscosity between 2.0 to $10 \mathrm{mPas}$ was reported as the acceptable viscosity for a continuous inkjet printing. ${ }^{17}$ Ink in $3 \mathrm{D}$ printing contains active pharmaceutical ingredient blended with polymers. ${ }^{5}$ Piezoelectric is the ability of an object to produce electricity in the presence of mechanical pressure. ${ }^{18} 3 \mathrm{D}$ inkjet printing can be divided into two types, namely Continuous Jet $(\mathrm{CJ})$ and Drop on Demand (DoD).

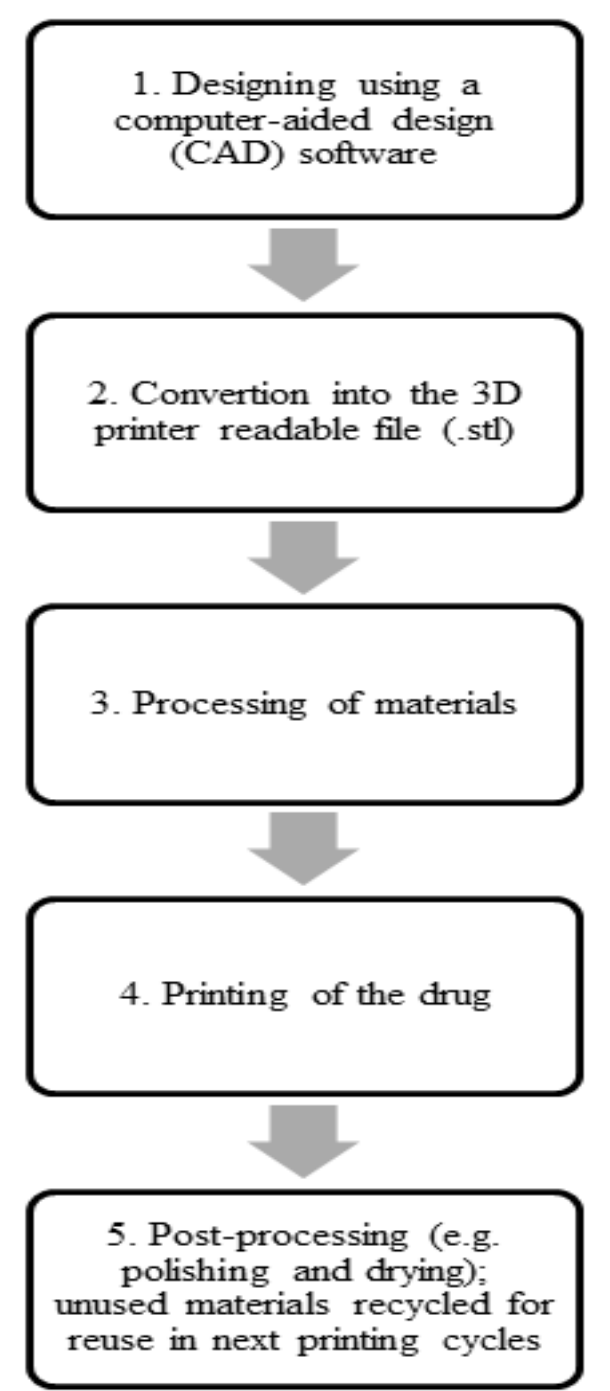

Figure 1: Basic procedure involved in the fabrication of 3D-printed drugs. ${ }^{15,16}$

\section{(i) Continuous Jet}

Continuous Inkjet $(\mathrm{CJ})$ incorporates pressure to drive the 'ink' liquid towards the nozzle tip for extrusion. As seen in Figure 2, a charged deflector at the nozzle tip serves to control the extrusion of 'ink' liquid either towards the printer stage for printing or waste chamber for recycling and reuse on the next printing. ${ }^{19,20}$

\section{(ii) Drop on Demand}

Drop on Demand (DoD) can be further classified into two types, which are thermal and piezoelectric. For thermal printing (Figure 2), the electrical pulse generates a rapid rise of heat to form a vapor bubble in the ink reservoir. The bubble then pushes the 'ink' out of the reservoir and onto the substrate before it collapses, thus producing negative pressure in the ink reservoir. ${ }^{1,21}$ The negative pressure causes any unused ink to refill the ink reservoir. As heat is utilized in this process, the material used as 'ink' must be non-volatile.

Piezoelectric printing (Figure 2) is characterized by the voltage that causes elements such as ceramics and crystals to deform. The deformation creates a pressure wave that mechanically extrudes fluid 'ink' from the nozzle onto the substrate. ${ }^{1}$ This method offers the advantage of thermo labile materials utilization as 'ink' as it does not implement heat usage. DOD is more advantageous than $\mathrm{CJ}$ as it is less wasteful and more precise, whereby it can extrude ink volume as low as $1-100$ pL per drop. ${ }^{1}$

\section{D Powder Bed Printing}

In this method, the liquid binding material is deposited onto a powder bed layer to bind the powder materials together and form a 3D object. The height of the powder bed layer is usually about $200 \mu \mathrm{m}$, while the size range of the powder bed particles is between 50 and 100

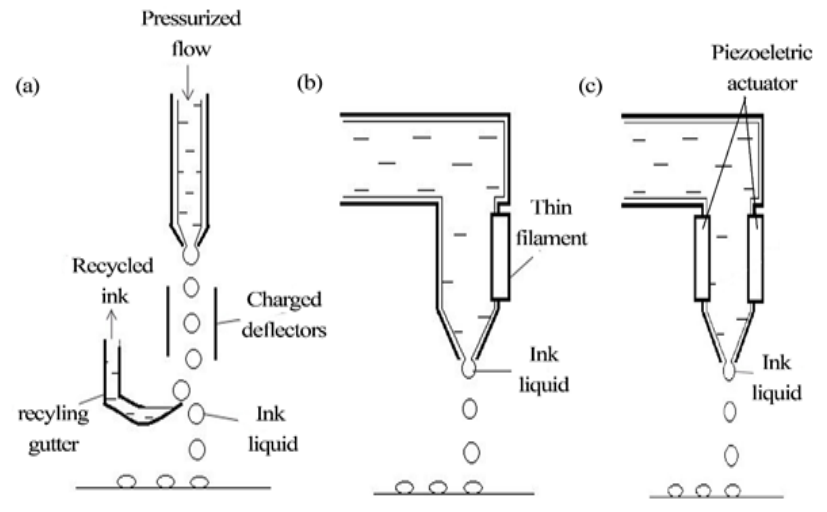

Figure 2: (a) Schematic Figure of a continuous jet printer. (b) Schematic Figure of Drop on Demand Printer. (c) Schematic Figure of Piezoelectric Printer. 
$\mu \mathrm{m}$. The first powder bed is lowered and another layer is distributed evenly on top using a roller, as shown in Figure 3. The same procedure is repeated continuously for a layer-by-layer generation until the desired object completes its fabrication. ${ }^{20}$

\section{Selective Laser Sintering (SLS)}

The concept of SLS is almost like 3D Powder Bed Printing. However, this method uses guided laser such as carbon dioxide as opposed to liquid to sinter the powder bed polymers and form a $3 \mathrm{D}$ project. As shown in Figure 4, the scanned laser increases the temperature of the powder bed polymers until the powder bed polymers reach the melting point. The polymer powder bed fuses as it melts, thus forming a 3D object. Then, a second layer is distributed evenly on the first powder layer, followed by the printing stage to be lowered for sintering to occur. The procedure is repeated until the desired object is fabricated. ${ }^{19,20}$

\section{Fused Deposition Modelling (FDM)}

FDM utilizes thermoplastic polymers such as polyvinyl acetate (PVA) in a semi-molten form rather than a powder bed. ${ }^{22}$ In this method, thermoplastic materials pass down between two rollers to the nozzle tip where it will be subsequently extruded (Figure 5). Before extrusion, the materials are heated by a temperaturecontrolled condition to ensure their semi-molten form during the extrusion process. Then, the semi-molten polymers solidify, the printing stage is lowered and the same process is repeated until the desired $3 \mathrm{D}$ object is obtained. ${ }^{19,20}$

\section{Pharmaceutical Applications of 3D-Printed Drugs Personalized Dosing}

Personalized medicine is defined as safe and effective customization of drug therapy dosing and delivery to consumers. ${ }^{23}$ The personalized dosing is significant as a patient response towards the same drug regimen varies across individuals. Therefore, the diversity of population results in some of them experiencing the

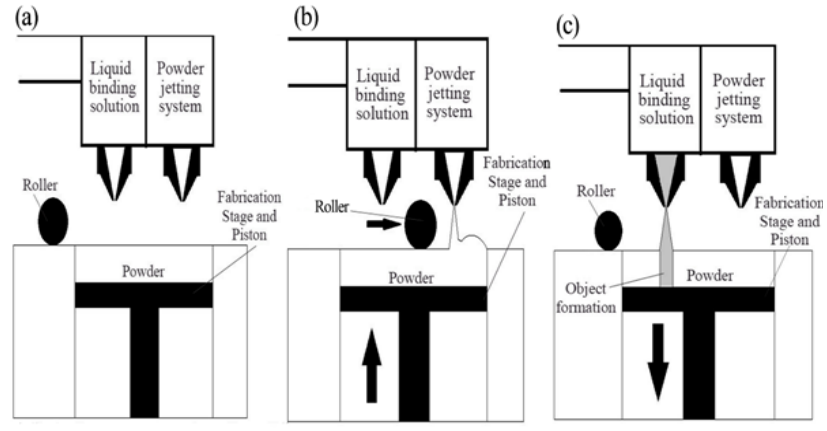

Figure 3: Schematic Figure of 3D Powder Bed Printer. desired therapeutic effect while the rest receive higher or lower doses than required, resulting in complaints of either side effects or ineffective treatment. Such concept is especially crucial for pediatric and geriatric patients. The needs of the former group are due to rapid changes in the development of their physiological and metabolic systems. In contrast, the needs for the latter group are due to pathologies affecting their gastrointestinal absorption, hepatic function, body fat and renal clearance. Besides, geriatric patients are further associated with polypharmacy and co-morbidities. Polypharmacy refers to multiple medicines usage due to co-morbidities; it is commonly associated with ADRs caused by drug-drug interactions or drugdisease interactions. ${ }^{13,23,24}$ Records show that on average, 65-year-old patients take 13 medicines, while some may consume as much as 28 medications concurrently. ${ }^{23}$ Therefore, their dosing regimen must be strictly controlled to reduce any potential interaction and ensure the effectiveness of the prescribed treatment.

Moreover, drugs solely available in single strength formulation is especially problematic for their administration to patients. Generally, crushing a solid tablet and measuring the volume of liquid medicine using measuring aids are the standard healthcare practices for such kind of drugs. However, these practices can result in dose variation and dose-dumping for enteric-coated tablets. ${ }^{25}$ The issue is particularly concerning in the case of drugs with a narrow therapeutic index and a small difference between blood therapeutic and toxic dose, whereby improper dosing leads to therapeutic failures or adverse side effects. ${ }^{16}$

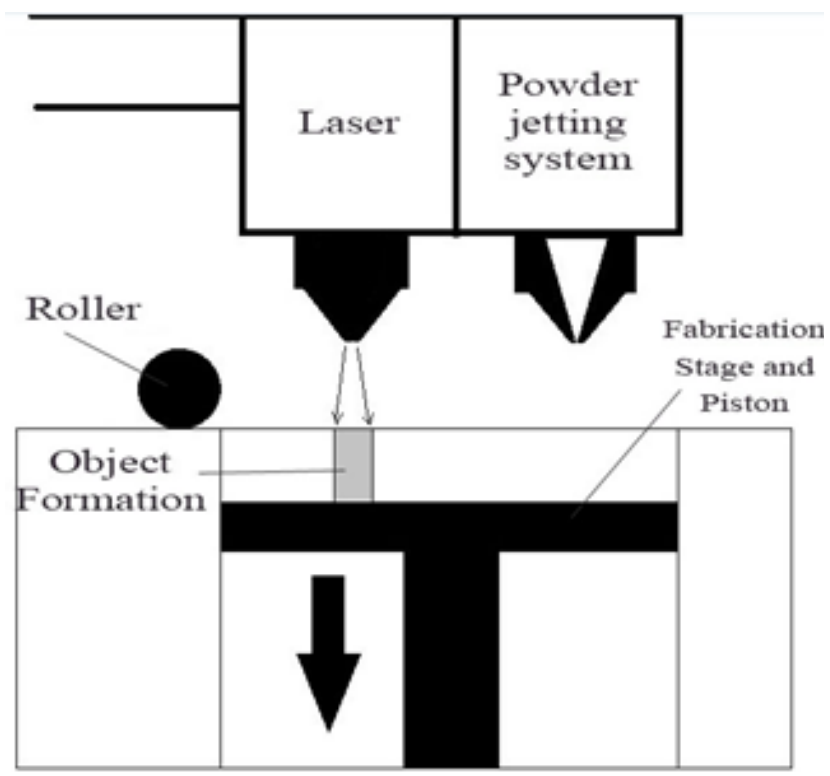

Figure 4: Schematic Figure of 3D Powder Bed Printer. 
The discussed issues may be overcome by inkjet printing which offers the potential of controlling a wide range of dose variations. The technology consequently optimizes drug dose accuracy and effectiveness of treatment, while also reducing drug wastage. ${ }^{26}$ The flexibility in controlling the deposited dose is an excellent approach to produce drugs for pediatric and geriatric patients and to produce personalized narrow therapeutic index drugs. ${ }^{27}$ Hence, issues such as medication errors, dose variations, ADRs and therapeutic failures can be avoided. Besides, 3D-printed drugs can simplify administration processes, thus resulting in improved patient compliance. ${ }^{16}$

Pharmaceutical-wise, 3D printing can produce a polypill, a single tablet that compartmentalizes several drugs, including incompatible drugs, into different regions of the tablet. Besides, the addition of polymers into the tablet ensure different drug release profiles. For instance, a study has been conducted incorporate two drugs in a single tablet, each of immediate and sustained release profiles, respectively. Such concept is demonstrated via guaifenesin, which is comprised of two different excipients that yield an immediate and sustained release of guaifenesin both in the single tablet. ${ }^{28}$

In another study, a sophisticated multi-compartment tablet containing three different APIs (i.e. captopril, glipizide and nifedipine) with different drug release profiles within the same single tablet has been fabricated. Captopril shows zero-order drug release in which the drug release is constant over time, whereas

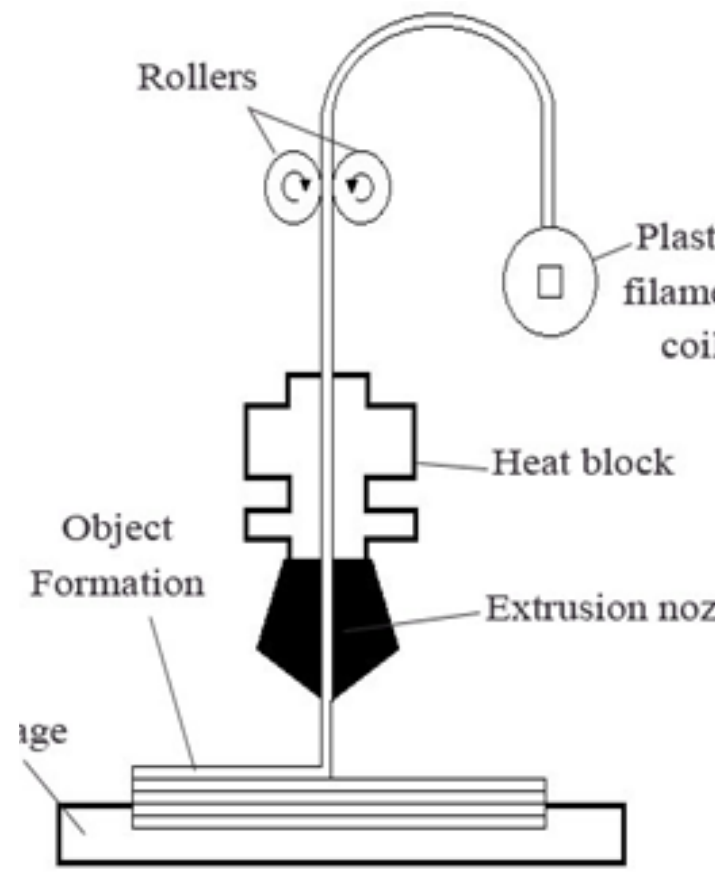

Figure 5: Schematic Figure of Fused Deposition Modelling. the remaining two drugs exert first-order drug release independent of the APIs concentration used. ${ }^{6}$

Similarly, Khaled et al. (2015b) have shown a successful fabrication of 3D-printed polypill, which consists of the five cardiac APIs of aspirin, hydrochlorothiazide, atenolol, Ramipril and pravastatin. The polypill is not only comprised of different APIs in a single tablet but also each varies in drug release profiles, which is beneficial to non-adherence patients. ${ }^{28}$

The drawback of the polypill concept is described as the more APIs added, the larger the tablet that will be formed. Such situation is not preferred among patients who have difficulty in swallowing. ${ }^{16}$ However, it can be overcome by varying the size of the 3D-printed tablet, which consequently adjusts the dose to be taken. ${ }^{13}$ Such alternative proves the flexibility dosing of the 3D-printed drug for personalized dosing without manipulating the drug formulation.

3D-printed drug application in personalized medicine is not limited to polypill usage only. It can also benefit those consuming drugs with a narrow therapeutic index, such as theophylline, methotrexate and the aminoglycosides. ${ }^{29,30}$ Pharmacists are responsible for analyzing the pharmacokinetics profile of the patient and the associated factors such as age, race and gender to optimize the drug dose. ${ }^{1}$

In the future, it is predicted that $3 \mathrm{D}$ printing will cause a paradigm shift transformation, from 'one-size-fits-all' to personalized medicine. Trenfield et al. (2018) have proposed for healthcare practitioners to be capable of quickly accessing and modifying the dosage of drugs based on real-time vital signs (e.g. heart rate and blood pressure), which can be monitored by the patients themselves using open applications on their smartphones. ${ }^{16}$ Based on the clinical response of specific patients, the dose of the drug may be adjusted later according to their needs (Figure 6 ).

\section{Variation of Drug Release Profile}

$3 \mathrm{D}$ printers can print a layer of the binder with a thickness of approximately $200 \mu \mathrm{m}$ onto substance as a barrier between the active ingredients. Different drug release profiles can be generated by printing layers between the active ingredients. ${ }^{19}$ Accordingly, 3D printers can fabricate complex geometries that are porous and loaded with multiple drugs surrounded by a barrier of layers, which control the drug release. ${ }^{19,20}$ Geometrical shapes, the porosity of the drug, drug concentration, polymer selection, the geometrical shapes of drugs are known to influence their release.

Goyanes et al. (2015) have studied the fabrication of five distinct drug shapes, namely cube, pyramid, 


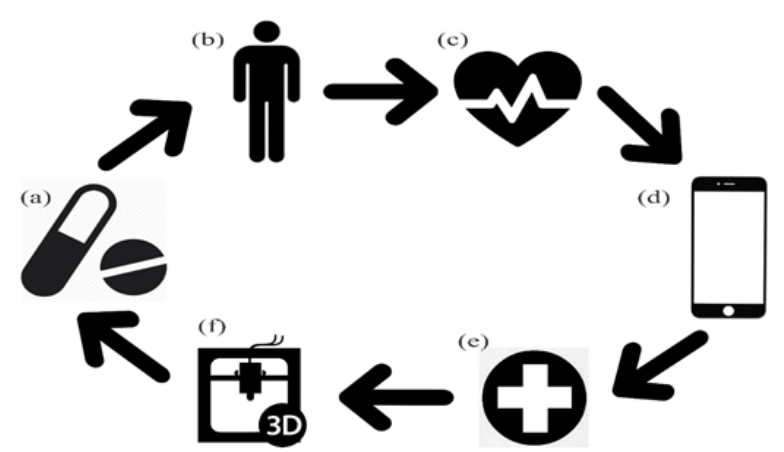

Figure 6: (a-b) Drugs are given to the patient. (b-c-d) Patients measure real-time vital signs (e.g. heart rate and blood pressure) using technologies such as a smartwatch or medical devices (e.g. sphygmomanometer), which are recorded in a computerized system (e.g. in smartphone). (e) Patient's health record is sent to healthcare practitioners to be monitored and intervened if dose adjustment is required. (f) If dose adjustment is required, the $3 \mathrm{D}$ printer can fabricate on-demand drugs based on the patient's needs (e.g. dose adjustment) and the adjusted 3D-printed drugs are dispensed to the patients.

cylinder, sphere and torus (doughnut like-shape); it is complicated and challenging to be achieved using conventional manufacturing methods. The drug dissolution is influenced by erosion-mediated process and dependent on the surface area over volume ratio rather than surface area alone. The study has shown that the torus shape is the most stable shape for drug release over time. By producing complex geometrical shapes of drugs, 3D-printed drug controls drug dissolution and renders it advantageous to be used to design a dosage form with different targeted sites in the gastrointestinal tract. $^{31}$

Overall, the potential of $3 \mathrm{D}$ printing to fabricate customized and personalized drug dosing, flexible dosage forms and novel drug release profiles could overcome major issues in the pharmaceutical field. Kook et al. (2012) also proved the same finding regarding the association between carrier shape or geometry to the drug release profile. Micro particles with 10\% (w/w) of paclitaxel-loaded poly (lactic-co-glycolic acid) (PLGA) was fabricated using piezoelectric inkjet printing into different shapes which are circles, grids, honeycombs and rings. Initially, the drug has burst release and subsequently followed by the slow release as the drug release depends on the surface area of the carrier. ${ }^{32}$ The research proved the capability of $3 \mathrm{D}$ printing to improve the precision and accuracy of drug release and delivery.

Another research finding has also pointed towards the stable dissolution of torus-shaped acrylonitrile butadiene styrene (ABS) scaffolds containing carbamazepine, which are fabricated by FDM. Only the area with holes

\begin{tabular}{|c|c|}
\hline Type of polymer & $\begin{array}{l}\text { Time taken for drug } \\
\text { disintegration (min) }\end{array}$ \\
\hline PVA-PEG & 60 \\
\hline HPC & 60 \\
\hline EC & Does not disintegrate \\
\hline HPMCAS & 420 \\
\hline
\end{tabular}

on the scaffolds undergoes dissolution, whereas the other parts of the scaffolds remain impermeable to release carbamazepine. The scaffold has thus demonstrated constant drug release and zero-order release kinetics. ${ }^{33}$ The research proves that complex geometries do affect overall drug release profile, which can minimize the frequency of drug dosing and indirectly improve patient compliance.

More recently, Xu et al. (2019) used the same concept, which was the application of fabricating different geometrical shapes using FDM to fabricate drugs with different drug release for various purposes. The study produced three geometrical shapes of paracetamol tablets which were cylinder, horn and reverse horn which achieved constant release, progressively increasing and progressively decreasing drug release, respectively. A consistent release drug profile is significant in ensuring a constant desired range of drug concentration is supplied, while gradually increasing drug release profile is applicable in case of drug resistance. ${ }^{34}$

A difference between conventional tablet compression and 3D-printed tablet is that the former uses an accurate spatial distribution of ingredients within a tablet. In contrast, the latter is not only able to produce as low as 10 to 12 moles of drugs per tablet but also position active ingredients within the tablet or dosage forms at will. ${ }^{22}$ With the capability to produce complex geometries and porous drug, as well as integrate drugs into the polymer base, 3D printing can vary the drug release profile.

Moreover, the drug amount and drug concentration variation in the homogenously polymer-modified base can affect the release rate and total release time of a 3D-printed drug. ${ }^{35}$ Herewith, the selection of a proper polymer influences the drug release profiles as proven by Goyanes et al. (2017). Their study has investigated a delayed-release tablet that can be produced by integrating the drug in an enteric polymer, which negates the need for an outer enteric shell. ${ }^{36}$

Aside from producing different drug release profile for the final tablet, 3D printing can also be used in the earlier 
stages of drug development to understand the drug release profiles. Gaisford et al. (2017) have demonstrated the drug release profiles of four different polymer-based devices, namely polyvinyl alcohol-polyethylene glycol graft-copolymer (PVA-PEG copolymer, Kollicoat ${ }^{\circledR}$ ); hydroxypropyl cellulose (HPC, Klucel ${ }^{\mathbb{}}$ ); ethyl cellulose (EC, Aqualon ${ }^{\circledR}$ N7); and hypromellose acetate succinate (HPMCAS, Aquasolve ${ }^{\circledR}-\mathrm{LG}$ ); which are made using 3D printer (Table 1). The difference in their drug release profiles has been observed in rats using Positron Emission Tomography/Computed Tomography (PET/ CT) imaging. The study has shown that it is advantageous to use the 3D-printed drug in observing drug release profiles in the earlier stages of drug development as opposed to the conventional manufacturing method to minimize waste, as the former manufacture small-scale products. $^{37}$

The tablet infill or drug porosity is also a critical aspect that can be modified to produce the drug at the desired rate of drug release. By applying these two concepts, Solanki et al. demonstrated a fabrication of immediate release haloperidol drugs with mixture of $1: 1 \mathrm{w} / \mathrm{w}$ of two polymers, polyvinylpyrrolidone-vinyl acetate copolymer (Kollidon ${ }^{\circledR}$ VA64) and hydroxypropyl methylcellulose HME 15 cP (HPMC HME 15 cP; Affinisol $\left.^{\mathrm{TM}} 15 \mathrm{cP}\right)$ since the polymers offered ideal mechanical strength to be produced by FDM. The percentage of tablet infill prepared were 100\% and 60\% and the $60 \%$ tablet infill showed a complete drug release in a much shorter time than the $100 \%$ tablet infill, which was in 45 min..$^{38}$

Another application for integrating polymers into 3D-printed drugs can be seen in oral drug delivery, which is particularly challenging as the absorption may vary due to factors like gastric secretions and gastric emptying. These conditions consequently lead to poor bioavailability and variation of serum drug concentration. ${ }^{13}$ However, this problem can be solved by increasing the gastric residence time, as it will increase the drug absorption and thus improving the bioavailability of oral drugs.

Furthermore, one of the ways to increase gastric residence time is the application of a floating tablet, which is achievable via either conventional drug production or $3 \mathrm{D}$ printing. The floating tablet releases drug content while floating on the surface of the gastric fluid, as it has less bulk density compared to the gastric fluid. ${ }^{35}$ However, the conventional drug production method is complicated and taxing for the optimization of the layer of multiple polymers.

HPC fabrication has been conducted for intragastric floating delivery of domperidone more economically by using FDM printers. A study has shown that the optimized formulation of $10 \%$ domperidone with two shells and $0 \%$ infill exerts sustained release drug profiles in about $10 \mathrm{hr}^{39}$ Based on the results, the ability of a drug to utilize long residence time in the gastrointestinal tract can contribute towards minimizing the frequency of drug consumption, as it can last for a longer duration of action.

Other studies have highlighted the fabrication of enteric-coated acid-sensitive drugs, namely budesonide for inflammatory bowel disease (IBD) treatment by using FDM printer. Firstly, budesonide is loaded with polyvinyl alcohol (PVA) caplets, whereby the caplets are then coated with a layer of enteric polymer using a bottom spray fluidized coater. ${ }^{13,40}$ The loading of drug with PVA and coating of caplet with bottom spray fluidized coater makes the drug becomes resistant towards the acidic environment of the stomach. The drug remains stable in the organ before disintegrating and releasing the active ingredients in the bowel due to its alkaline environment.

Apart from slow-release drugs, 3D printing can also fabricate immediate release 3D-printed drugs, which is particularly useful for fabricating analgesic drugs. Hydrophilic pharmaceutical-grade polymers such as polyvinyl pyrrolidone (PVP) and Eudragit ${ }^{\circledR}$ EPO (expanded Polyolefin) has been fabricated into the active ingredients to produce immediate-release medication while in contact with water using SLS and FDM. ${ }^{36,41,42}$

Moreover, another way to fabricate immediate release drug is by producing porous drugs as possibly done by the 3D printer. ${ }^{43}$ Generally, 3D-printed drugs are more porous and friable. Aprecia Pharmaceuticals has implemented the feature in their powder bed printing technology to manufacture and patent Zip Dose ${ }^{\circledR}$, an orodispersible tablet that rapidly dissolves within 10 sec in $15 \mathrm{~mL}$ or less amount of water. Following this, it has led to the production and approval of the first 3D-printed drug Spritam $^{\circledR}$ (levetiracetam) for antiepilepsy, especially for those with difficulty in swallowing tablets, such as pediatric and geriatric patients. ${ }^{1}$

On top of oral drug delivery, 3D printing can modulate drug release in other dosage forms, such as implants for bone infection treatment. Implants are drug loadeddevice installed on the body surface or into the body to deliver the drug at the desired rate. ${ }^{44}$ Powder-based 3D-printed bone scaffolding manufacturing is possible in high-resolution models with macrospores as small as $0.5 \mathrm{~mm}$, complex geometries and microporosity, which are collectively crucial for fluid exchange and cellular influx during bone healing. ${ }^{45}$ 
Furthermore, the 3D printing technology fabricates the specific geometries for the patient and control the spatial distribution of active ingredients and polymers within the scaffold to modulate the drug release profiles. ${ }^{46}$ Predefined microstructures enable the complex and tailored medicine such as 3D printed levofloxacin implants to exert both pulsatile and constant drug release. ${ }^{47}$

Successful fabrication of different percentage of nitrofurantoin and hydroxyapatite loaded implants by Water et al. (2015) suggested that the release of drugs depends on the percentage of drug loads; drugs release is higher in the higher percentage of the drugs load. ${ }^{48}$ All these applications are almost impossible or hard to be achieved by using the conventional method to fabricate 3D-printed bone implants.

Another study has depicted bone-implant consisting of two drugs, namely levofloxacin and rifampicin, with the immediate release of levofloxacin on the first day and delayed release of rifampicin on the eighth day. Both drugs are made for sustained release for six weeks in total. ${ }^{1}$ The application proves the capability of the $3 \mathrm{D}$ printer; it not only incorporates different drugs together but also modulates the multi-mechanism of release profiles.

\section{Flexible Dosage Form}

3D printing may potentially create limitless varieties of dosage form, which is an initiative to improve patient acceptability towards drug consumption. It incorporates the elements of ease of ingestion, such as palatability, swallow ability, smaller size and quantity of solid dosage forms. ${ }^{49}$ Similarly, the variety of dosage forms aims to ease drug administration, particularly those of oral dosage forms. Although liquid formulations are available for those with swallowing difficulties, such formulation can be non-preferable due to strong medicinal taste, low stability of the drug and the inconvenience of measuring doses compared to solid dosage forms. ${ }^{13}$

Therefore, the problem can be overcome by fabricating orodispersible tablets, which as solid tablets that disperse in the oral cavity. Spritam ${ }^{\circledR}$ is an anti-epilepsy that is the first FDA-approved 3D-printed drug capable of dispersing in mere seconds due to the incorporation of levetiracetam to soluble matrix composition. It is also due to its porous structure as there is a lack of compression forces in Spritam ${ }^{\circledR}$ compared to the conventional compressed tablet. ${ }^{14,50}$ Some of the limitations of conventional compressed tablet include: the dissolution time, their facility to be swallowed, and the dosage strengths that may be accommodated. Spritam ${ }^{\circledR}$ is produced using $3 \mathrm{D}$ printing technology to overcome these limitations by a novel, porous, quickly disintegrating, and easier-to-swallow due to fast-melt based on powder-liquid formulation.

Other research findings have applied the same concept of orodispersible tablets but by using paracetamol as the active ingredient, which is fabricated with watersoluble thermoplastic PVA. The formulation causes the tablets to dissolve readily in the oral cavity, particularly for thin films. ${ }^{51}$ All in all, fast disintegrating tablet in an oral cavity facilitates the swallowing action, especially for children, elderly patients and those having difficulties in swallowing.

Besides the ease of swallowing, another issue about patient's acceptability, particularly among pediatric patients, is that they usually do not like to consume medication. This problem can be solved by making the dosage forms more attractive and presentable using the Starmix ${ }^{\circledR}$ design of tablets, which consists of the various shapes of heart, ring, bottle, bear and lion-shaped solid dosage forms. ${ }^{52}$

Recently, Scoutaris et al. (2018) have implemented the concept by fabricating indomethacin with hypromellose acetate succinate (HCMCAS) and polyethylene glycol (PEG) by using hot-melt extrusion (HME) coupled with FDM. The drug filament has been successfully processed by the 3D printer to fabricate the desired shapes according to the Starmix ${ }^{\circledR}$ design. ${ }^{52}$ This drug targets pediatric patients to consume them, thus ultimately improving the compliance problem.

Moreover, 3D printer is capable of fabricating dosage forms other than for oral administration, such as 3D transdermal delivery system (TDS). ${ }^{15}$ The advantages of the dosage form include its avoidance of first-pass metabolism and $\mathrm{pH}$-mediated degradation, which eases drug administration for those with chronic diseases such as diabetes mellitus (DM). According to Prasad et al. (2016), 3D printer fabricates drug-loaded microneedles with a height of about less than $500 \mu \mathrm{m}$, which are intended to penetrate the stratum corneum $(10-15 \mu \mathrm{m})$ and deliver the drugs accordingly. ${ }^{1}$

Theoretically, the microneedles should possess enough strength to penetrate the epidermis. However, if they are too strong, they may result in pain or irritation upon application. Besides, biodegradable polymers such as poly(methyl vinyl ether-alt-maleic anhydride) are recommended to be used in fabricating the microneedles as a safety measure in case the tip breaks off and gets impregnated in the skin., ${ }^{1,53}$ Interestingly, 3D printers can fabricate such complex dosage forms 
even with the intricate geometries of sizes and shapes of microneedles. ${ }^{52}$

\section{Limitations and Challenges}

The implementation of $3 \mathrm{D}$ printing technologies to produce personalized drugs, the variation of drug release profile and unique dosage forms have been gradually increased. However, there are also limitations and challenges faced by this technology in drugs production. Some processes in $3 \mathrm{D}$ printing may not be suitable for some active ingredients or polymers due to the destructive or degradation caused by the method. For instance, the FDM method utilizes heat in the process; thus, this method is not applicable to produce heatlabile drugs such as biomolecules like protein. ${ }^{14,19,20}$ Dose adjustment is frequently based on empirical methods, and therefore, the chance of undesirable side effects to occur is high. Comprehensive research must be conducted to select a suitable 3D printing method to fabricate a specific drug based on the nature of the active ingredients. The appropriate method used will preserve the quality of drug present in the product fabricated, hence ensuring the efficacy of drug delivery. Another limitation of 3D printing for drug manufacturing is that this method is challenging to scale-up compared to the conventional manufacturing method. Other than for rapid fabrication of drugs prototype, $3 \mathrm{D}$-printing is primarily used for fabrication of personalized drugs or drugs with unique characteristics, which the fabrication process is conducted in smallscale. However, the current 3D-printing process is not suitable for the more substantial scale of drug manufacturing due to the slower process as than the conventional manufacturing method. The conventional manufacturing methods are easier to increase the size of production as the processes involve such as tablet compaction can be conducted at high speed. ${ }^{13}$

Application of 3D-printing in drug manufacturing also faces some issues concerning regulatory requirements by the FDA. Currently, there is no specific guideline regarding 3D-printing for drug manufacturing. ${ }^{13,54,55}$ Although the FDA approved Spritam ${ }^{\circledR}$, the method used to produce larger yield production for this product was actually about the same as conventional tableting method rather than using 3D-printing technologies. ${ }^{13}$ Also, there are challenges relating to protection intellectual right

\section{CONCLUSION}

The 3D-printed drug is the drug manufactured layerby-layer to produce a complex personalized drug on demand. The technology offers better drug spatial distributions, more accurate drug dosing extrusion and complex geometries compared to conventional drugs. These advantages lead to the rapid increase of 3D printing applications since researchers are gradually and consistently improving the performance, resolution and overall applications of $3 \mathrm{D}$ printing in the pharmaceutical field. The approval of Spritam ${ }^{\circledR}$ anti-epileptic by FDA in 2015 sparked researchers' concentrated efforts further to improve the applications of $3 \mathrm{D}$ printing in the field. Besides, 3D printing covers a wide range of applications, from the stage of drug development in the pre-clinical trials up until the last stage of drug dispensing in pharmacy or hospital settings. Overall, the potential of $3 \mathrm{D}$ printing to fabricate customized and personalized drug dosing, flexible dosage forms and novel drug release profiles can overcome major issues in the pharmaceutical field, such as patient compliance, medication non-adherence, ineffective treatment due to sub-therapeutic drug dosing and ADRs after medication overdose.

\section{ACKNOWLEDGEMENT}

Ministry of Education Malaysia financially supported this review paper under FRGS RACER grant:RACER/1/2019/SKK09/UIAM//2.

\section{CONFLICT OF INTEREST}

The authors declare that there is no conflict of interest.

\section{ABBREVIATIONS}

3D: Three-dimesional; AM: Additive Manufacturing; FDA: Food and Drug Administration; CAD: Computer-aided Design; APIs: Active Pharmaceutical Ingredients; ADR: Adverse Drug Reaction; SLS: Selective Laser Sintering; FDM: Fused Deposition Modelling; CJ: Continuous Jet; DoD: Drop on Demand; PVA: Polyvinyl acetate; PLGA: Poly (lacticco-glycolic acid); ABS: Acrylonitrile Butadiene Styrene; PVA-PEG: Polyvinyl alcohol-polyethylene glycol; HPC: Hydroxypropyl cellulose; EC: Ethyl cellulose; HPMCAS: Hypromellose acetate succinate; PET/ CT: Positron Emission Tomography/Computed Tomography; HPMC: hydroxypropyl methylcellulose; IBD: Inflammatory Bowel Disease; PVP: Polyvinyl Pyrrolidone; EPO: Expanded Polyolefin; HME: Hot Melt Extrusion; TDS: Transdermal Delivery System; DM: Diabetes Mellitus. 


\section{REFERENCES}

1. Prasad LK, Smyth H. 3D Printing technologies for drug delivery: A review. Drug Development and Industrial Pharmacy. 2016;42(7):1019-31. doi:10.31 09/03639045.2015.1120743

2. Awad A, Trenfield SJ, Goyanes A, Gaisford S, Basit AW. Reshaping drug development using 3D printing. Drug Discovery Today. 2018;23(8):1547-55. doi:10.1016/j.drudis.2018.05.025

3. Attaran $M$. The rise of 3-D printing: The advantages of additive manufacturing over traditional manufacturing. Business Horizons. 2017;60(5):677-88. doi:10.1016/j.bushor.2017.05.011

4. Lepowsky E, Tasoglu S. 3D printing for drug manufacturing: A perspective on the future of pharmaceuticals. International Journal of Bioprinting. 2018;4(1):119.

5. Lamichhane S, Bashyal S, Keum T, et al. Complex formulations, simple techniques: Can 3D printing technology be the Midas touch in pharmaceutical industry?. Asian Journal of Pharmaceutical Sciences. 2019;14(5):465-79. doi:10.1016/j.ajps.2018.11.008

6. Khaled SA, Burley JC, Alexander MR, Yang J, Roberts CJ. 3D printing of tablets containing multiple drugs with defined release profiles. International Journal of Pharmaceutics. 2015;494(2):643-50. doi:10.1016/j. ijpharm.2015.07.067

7. Jamróz W, Szafraniec J, Kurek M, Jachowicz R. 3D Printing in Pharmaceutical and Medical Applications: Recent Achievements and Challenges. Pharmaceutical Research. 2018;35(9):176.

8. Sandler N, Preis M. Printed Drug-Delivery Systems for Improved Patient Treatment. Trends in Pharmacological Sciences. 2017;38(3):317. doi:10.1016/j.tips.2016.10.002

9. Rajesh, Agrawal, Naveen Y. Pharmaceutical Processing: A Review on Wet Granulation Technology. 2011;1(1):65-83.

10. Veeren JC, Weiss M. Trends in emergency hospital admissions in England due to adverse drug reactions: 2008-2015. Journal of Pharmaceutical Health Services Research. 2017;8(1):5-11. doi:10.1111/jphs.12160

11. Chan SL, Ang X, Sani LL, et al. Prevalence and characteristics of adverse drug reactions at admission to hospital: A prospective observational study. British Journal of Clinical Pharmacology. 2016;82(6):1636-46. doi:10.1111/ bcp. 13081

12. Shepherd G, Mohorn P, Yacoub K, May DW. Adverse Drug Reaction Deaths Reported in United States Vital Statistics, 1999-2006. Annals of Pharmacotherapy. 2012;46(2):169-75. doi:10.1345/aph.1P592

13. Lim SH, Kathuria H, Tan JJY, Kang L. 3D printed drug delivery and testing systems: A passing fad or the future?. Advanced Drug Delivery Reviews. 2018;132:139-68. doi:10.1016/j.addr.2018.05.006

14. Konta A, García-Piña M, Serrano D. Personalised 3D Printed Medicines: Which Techniques and Polymers Are More Successful?. Bioengineering. 2017;4(4):79. doi:10.3390/bioengineering4040079

15. Norman J, Madurawe RD, Moore CMV, Khan MA, Khairuzzaman A. A new chapter in pharmaceutical manufacturing: 3D-printed drug products. Advanced Drug Delivery Reviews. 2017;108:39-50. doi:10.1016/j. addr.2016.03.001

16. Trenfield SJ, Awad A, Goyanes A, Gaisford S, Basit AW. 3D Printing Pharmaceuticals: Drug Development to Frontline Care. Trends in Pharmacological Sciences. 2018;39(5):440-51. doi:10.1016/j. tips.2018.02.006

17. Liu Z, Zhang M, Bhandari B, Wang Y. 3D printing: Printing precision and application in food sector. Trends in Food Science and Technology. 2017. doi:10.1016/j.tifs.2017.08.018

18. Manbachi A, Cobbold RSC. Review Development and application of piezoelectric materials for ultrasound generation and detection. Ultrasound. 2011;19(4):187-96.

19. Ventola CL. Medical Applications for 3D Printing: Current and Projected Uses. $\mathrm{P}$ and $\mathrm{T}$ : A Peer-reviewed Journal for Formulary Management. 2014;39(10):704-11. doi:10.1016/j.infsof.2008.09.005

20. Gross BC, Erkal JL, Lockwood SY, Chen C, Spence DM. Evaluation of 3D Printing and Its Potential Impact on Biotechnology and the Chemical Sciences. Analytical Chemistry. 2014;86(7):3240-53. doi:10.1021/ac403397r

21. Murphy SV, Atala A. Review 3D bioprinting of tissues and organs. Nature Publishing Group. 2014;32(8):773-85. doi:10.1038/nbt.2958
22. Alhnan MA, Okwuosa TC, Sadia M, Wan KW, Ahmed W, Arafat B. Emergence of 3D Printed Dosage Forms: Opportunities and Challenges. Pharmaceutical Research. 2016;33(8):1817-32. doi:10.1007/s11095-016-1933-1

23. Masnoon N, Shakib S, Kalisch-Ellett L, Caughey GE. What is polypharmacy? A systematic review of definitions. BMC Geriatrics. 2017;17(1):1-10. doi:10.1186/s12877-017-0621-2

24. Maher RL, Hanlon J, Hajjar ER. Clinical consequences of polypharmacy in elderly. Expert Opinion Drug Safety. 2014;13(1):57-65. doi:10.1517/147403 38.2013.827660

25. Habib WA, Alanizi AS, Alanizi FK. Accuracy of tablet splitting: Comparison study between hand splitting and tablet cutter. Saudi Pharmaceutical Journal. 2014;22(5):454-9. doi:10.1016/j.jsps.2013.12.014

26. Alomari M, Mohamed FH, Basit AW, Gaisford S. Personalized dosing: Printing a dose of one's own medicine. International Journal of Pharmaceutics. 2015;494(2):568-77. doi:10.1016/j.ijpharm.2014.12.006

27. Vakili H, Kolakovic R, Genina N, et al. Hyper spectral imaging in quality control of inkjet printed personalized dosage forms. International Journal of Pharmaceutics. 2015;483(1-2):244-9. doi:10.1016/j.jpharm.2014.12.034

28. Khaled SA, Burley JC, Alexander MR, Yang J, Roberts CJ. 3D printing of five-in-one dose combination polypill with defined immediate and sustained release profiles. Journal of Controlled Release. 2015;217:308-14. doi:10.1016/j.jconrel.2015.09.028

29. Pietrzak K, Isreb A, Alhnan MA. A flexible-dose dispenser for immediate and extended release 3D printed tablets. European Journal of Pharmaceutics and Biopharmaceutics. 2015;96(8):380-7. doi:10.1016/j.ejpb.2015.07.027

30. Weisman JA, Nicholson JC, Tappa K, Jammalamadaka U, Wilson CG, Mills D. Antibiotic and chemotherapeutic enhanced three-dimensional printer filaments and constructs for biomedical applications. International Journal of Nanomedicine. 2015;10:357-70. doi:10.2147/IJN.S74811

31. Goyanes A, Robles Martinez P, Buanz A, Basit AW, Gaisford S. Effect of geometry on drug release from $3 \mathrm{D}$ printed tablets. International Journal of Pharmaceutics. 2015;494(2):657-63. doi:10.1016/j.ijpharm.2015.04.069

32. Kook B, Hee Y, Suk J, Chan Y, Dong J, Woo Y. Fabrication of drug-loaded polymer microparticles with arbitrary geometries using a piezoelectric inkjet printing system. International Journal of Pharmaceutics. 2012;427(2):305-10. doi:10.1016/j.ijpharm.2012.02.011

33. Lim SH, Chia SMY, Kang L, Yap KYL. Three-Dimensional Printing of Carbamazepine Sustained-Release Scaffold. Journal of Pharmaceutical Sciences. 2016;105(7):2155-63. doi:10.1016/j.xphs.2016.04.031

34. Xu X, Zhao J, Wang M, Wang L, Yang J. 3D Printed Polyvinyl Alcohol Tablets with Multiple Release Profiles. Scientific Reports. 2019;9(1):12487. doi:10.1038/s41598-019-48921-8.

35. Gharti KP, Budhathoki U, Thapa P, Bhargava A. Formulation in vitro evaluation of floating tablets of hydroxypropyl methylcellulose and polyethylene oxide using ranitidine hydrochloride as a model drug. Journal of Young Pharmacists. 2012;4(4):201-8. doi:10.4103/0975-1483.104363

36. Goyanes AA, Fina F, Martorana A, Sedough D, Gaisford S, Basit AW. Development of modified release $3 D$ printed tablets (printlets) with pharmaceutical excipients using additive manufacturing. International Journal of Pharmaceutics. 2017;527(1-2):21-30. doi:10.1016/j.ijpharm.2017.05.021

37. Gaisford S, Aguiar P, Abdul W. PET/CT imaging of $3 \mathrm{D}$ printed devices in the gastrointestinal tract of rodents. International Journal of Pharmaceutics. 2017;536(1):158-64. doi:10.1016/j.ijpharm.2017.11.055

38. Solanki N, Shah A, Serajuddin ATM. Formulation of 3D Printed Tablet for Rapid Drug Release by Fused Deposition Modeling (FDM): Screening Polymers for Drug Release, Drug-Polymer Miscibility and Printability. Journal of Pharmaceutical Sciences. 2017. doi:10.1016/j.xphs.2017.10.021

39. Chai X, Chai H, Wan X, et al. Fused Deposition Modeling ( FDM ) 3D Printed Tablets for Intragastric Floating Delivery of Domperidone. Scientific Report. 2017;7(2):1-9. doi:10.1038/s41598-017-03097-x

40. Goyanes A, Chang $H$, Sedough D, et al. Fabrication of controlled-release budesonide tablets via desktop (FDM) 3D printing. International Journal of Pharmaceutics. 2015;496(2):414-20. doi:10.1016/j.jpharm.2015.10.039

41. Okwuosa TC, Stefaniak D, Arafat B, Isreb A, Wan K. A Lower Temperature FDM 3D Printing for the Manufacture of Patient-Specific Immediate Release Tablets. Pharmaceutical Research. 2016;33(11):2704-12. doi:10.1007/ s11095-016-1995-0 
42. Sadia M, Sośnicka A, Arafat B, et al. Adaptation of pharmaceutical excipients to FDM 3D printing for the fabrication of patient-tailored immediate release tablets. International Journal of Pharmaceutics. 2016;513(1-2):659-68. doi:10.1016/j.jpharm.2016.09.050

43. Khaled SA, Burley JC, Alexander MR, Roberts CJ. Desktop 3D printing of controlled release pharmaceutical bilayer tablets. International Journal of Pharmaceutics. 2014;461(1-2):105-11. doi:10.1016/j.ijpharm.2013.11.021

44. Shende P, Agrawal S. Biomedicine and Pharmacotherapy Integration of 3D printing with dosage forms: A new perspective for modern healthcare. Biomedicine and Pharmacotherapy. 2018;107(7):146-54. doi:10.1016/j. biopha.2018.07.167

45. Inzana JA, Olvera D, Fuller SM, et al. 3D printing of composite calcium phosphate and collagen scaffolds for bone regeneration. Biomaterials. 2014;35(13):4026-34. doi:10.1016/j.biomaterials.2014.01.064

46. Ozbolat I, Yu Y. Bioprinting towards Organ Fabrication: Challenges and Future Trends. IEEE Transactions on Biomedical Engineering. 2013;60(3):691-9.

47. Huang $W$, Zheng $Q$, Sun $W, X u H$, Yang X. Levofloxacin implants with predefined microstructure fabricated by three-dimensional printing technique. International Journal of Pharmaceutics. 2007;339(1-2):33-8. doi:10.1016/j. ijpharm.2007.02.021

48. Water JJ, Bohr A, Boetker J, et al. Three-Dimensional Printing of Drug-Eluting Implants: Preparation of an Antimicrobial Polylactide Feedstock Material. Journal of Pharmaceutical Sciences. 2015;104(3):1099-107. doi:10.1002/ jps. 24305

49. Walsh J, Ranmal SR, Ernest TB, Liu F. Patient acceptability, safety and access: A balancing act for selecting age-appropriate oral dosage forms for paediatric and geriatric populations. International Journal of Pharmaceutics. 2018;536(2):547-62. doi:10.1016/j.jpharm.2017.07.017

50. Boudriau S, Hanzel C, Massicotte J, Sayegh L, Wang J, Lefebvre M. Randomized Comparative Bioavailability of a Novel Three-Dimensional Printed Fast-Melt Formulation of Levetiracetam Following the Administration of a Single 1000-mg Dose to Healthy Human Volunteers Under Fasting and Fed Conditions. Drugs in R and D. 2016;16(2):229-38. doi:10.1007/s40268016-0132-1

51. Yu DG, Branford-White C, Yang YC, Zhu LM, Welbeck EW, Yang XL. A novel fast disintegrating tablet fabricated by three-dimensional printing. Drug Development and Industrial Pharmacy. 2009;35(12):1530-6. doi:10.3109/03639040903059359

52. Scoutaris N, Ross SA, Douroumis D. 3D Printed "Starmix" Drug Loaded Dosage Forms for Paediatric Applications. Pharmaceutical Research. 2018;35(2):34.

53. Boehm RD, Miller PR, Hayes SL. Modification of microneedles using inkjet printing Modification of microneedles using inkjet printing. AIP Advances. 2011;1(2):022139. doi:10.1063/1.3602461

54. Bhusnure OG, Gholve S V, Sugave BK, Dongre RC, Gore SA, Giram PS. 3D Printing and Pharmaceutical Manufacturing: Opportunities and Challenges. International Journal of Bioassays. 2016;5(1):4723-38. doi:10.21746/ ijbio.2016.01.006

55. Jose A. 3D Printing of Pharmaceuticals: A Potential Technology in Developing Personalized Medicine. Asian Journal of Pharmaceutical Research and Development. 2018;6(3):46-54.

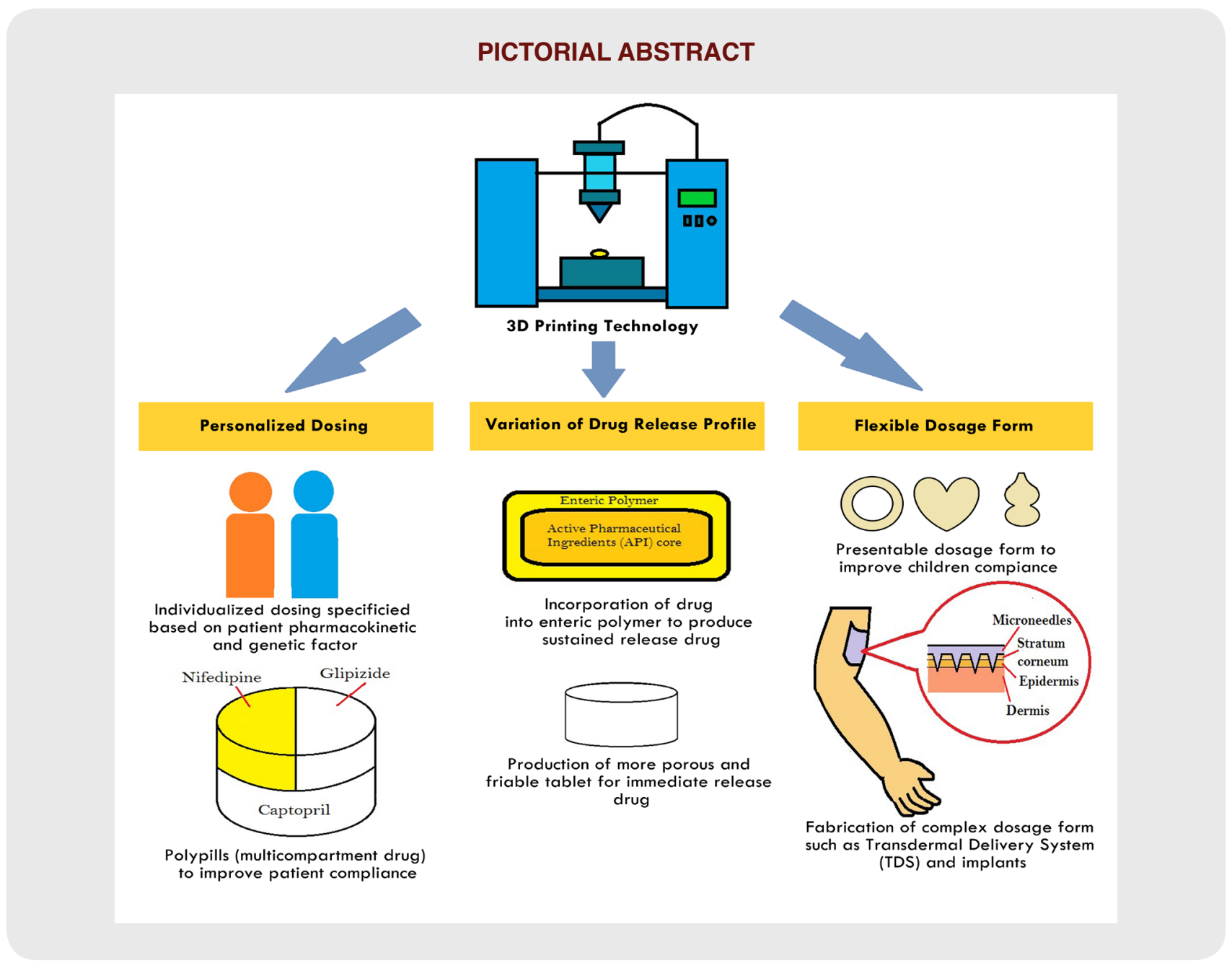




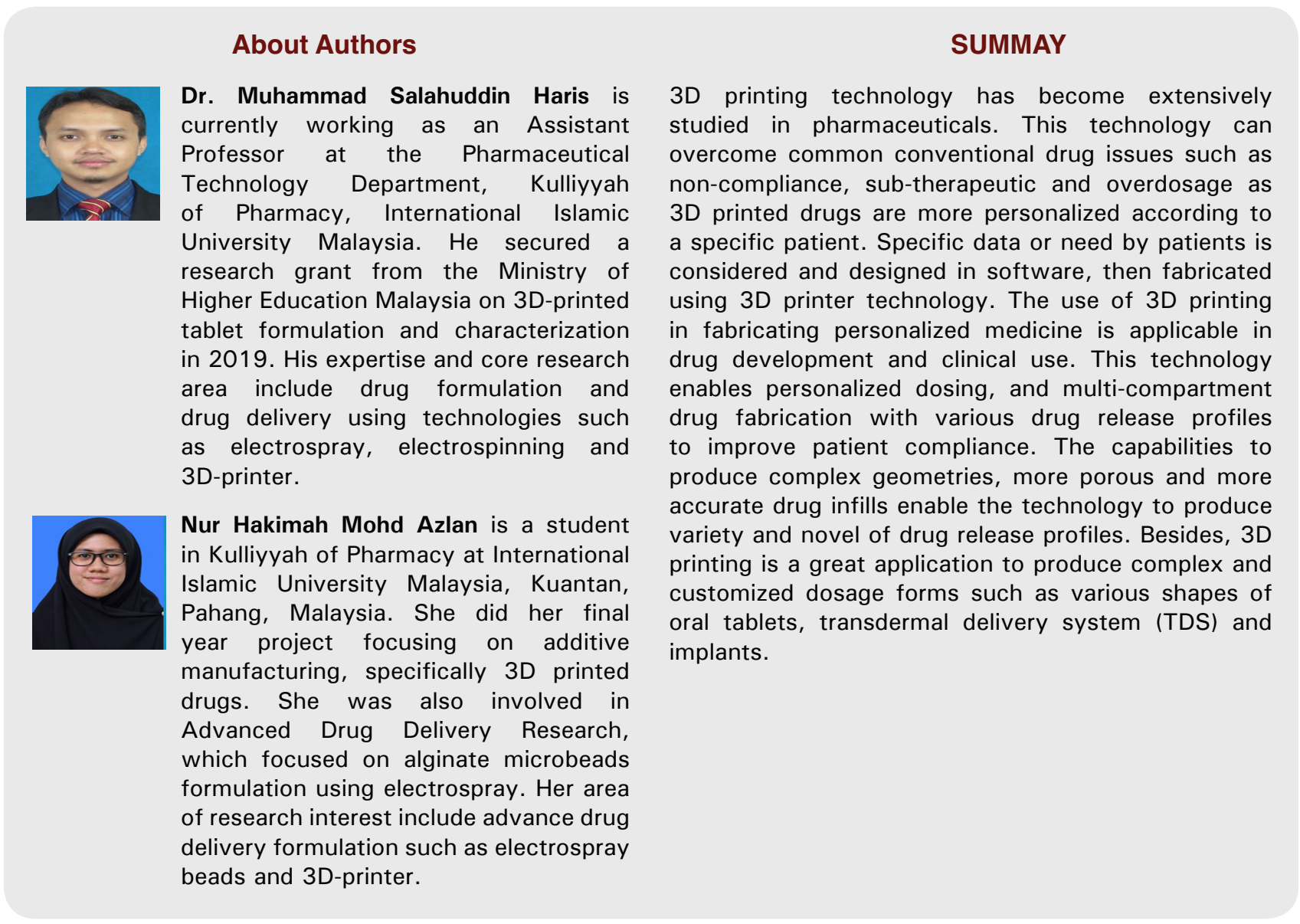

Cite this article: Haris MS, Azlan NHM, Taher M, Rus SM, Chatterjee B. 3D-printed Drugs: A Fabrication of Pharmaceuticals towards Personalized Medicine. Indian $\mathrm{J}$ of Pharmaceutical Education and Research. $2020 ; 54(3 s): s 411-s 422$. 